Gynäk. Rdsch. 1979;19(Suppl. 2):I-IV

\title{
Contents, Vol. 19, Supplement 2, 1979
}

1. Haupithema

Psychologie und Perinatalmedizin

1 er sujet principal

Psychologie et médecine pérínatale

Moderator - Modérateur: J. Bretscher, Zurich

Bretscher, J.: Einfúhrung

Kruse, F.: Spekulationen und beweisbare Tatsachen der pränatalen Psychologie ....

Hirsch, H.A.: Risiken der Klinikgeburt gegenüber der Hausgeburt $\quad 18$

Bretscher, J.: Programmierte Geburt

Schwammberger, R.: Verunsicherung der Frauen durch die Massenmedien

Bretscher, J.: Schlussbemerkungen $\quad 30$

Mitieilungen zum 1. Haupithema

Communications se rapportant au ler sujet principal

Morf, P. und Morf, U.: Beeinflussung des ersten Mutter-Kind-Kontaktes durch geburtshilfliche Massnahmen

31

Ammann, K. und Elsässer, R.: Die familienbezogene Geburtshilfe in ihrer Praxis ....

Landolt, A.; Vetter, K. und Siegrist, J.: Erlebnis von Geburt und Wochenbett an einer

Universitätsklinik. Vorläufige Ergebnisse einer Umfrage 34

Dayer, A.M.; Wyss, R. et Béguin, F.: Grossesse et accouchement chez Tadolescente . .

Index II

2. Hauptthema Gynäkologie der alien Frau

2e sujet principal Gynécologie du troisième age

Junod, J.-P.: Principes et limites de Faction gériatrique

39

Eisdorfer, C. and Wilkie, F.L.: Stress and Behavior in the Aged

Robert, H.G.: L'incontinence urinaire en géronto-gynécologie

45

57

Krauer, F.: La chirurgie gynécologique chez les octogénaires. Indications - selection limites $\quad 66$

Rifat, K.: Anesthésie-réanimation des personnes âgées

Mitteilungen zum 2. Hauptthema Communications se rapportant au 2e sujet principal

Walther, M. und Gigon, U.: Operative gynäkologische Geriatrie der Universitäts-

Frauenklinik Bern der Jahre 1976/1977 77

Derungs, R. und Morf, P.: Morbidität bei vaginalen Hysterektomien und Prolapsoperationen bei über 70jährigen Frauen 79

Fischer, B. von; Dreher, E.; Adam, P.J. und Maurer, H.P.: Erfahrungen mit der medikamentösen Behandlung von Urin- und Stuhlinkontinenz in der Postmenopause 80

Levy, Ph.; Krauer, F. et Junod, J.P.: La femme âgée et le cancer du sein: queue attitude adopter? 83

Obolensky, W.: Die Labiensynechie 84 
Freie Mitteilungen Communications libres

Perinatologie/Geburtshilfe Périnatologie/Obstétrique

Jann, F.X.;Dreher, E. und Locher, P.: Antibiotikaprophylaxe bei Sectio caesarea . ... 86 Käch, O.;

Senn, C. und Lüscher, P.: Beckenendlage und ihre Azidosemorbidität.

Analyse anhand von 258 Fallen $\quad 88$

Rageth, S.; Reubi, F.; Endtner, B. und Flückiger, J.: Schwangerschaft nach Blasen-

ekstrophie $\quad 90$

Bretscher, J.; Hoffmann, M.; Stepanic, V. und Stoffel, R.: Ultraschalldiagnostik der

angeborenen Halszyste und des Kleeblattschädels 91

Schams, H.: Mütterlicher Tod infolge Ileus in Graviditate in der 32. Schwangerschaftswoche 93

Sidiropoulos, D.; Jann, F.X.; Gigon, U. und Auf der Maur, P.: Resultate der pränatalen Diagnostik nach Fruchtwasseruntersuchungen bei 638 Fallen $\quad 95$

Index III

Gynäkologische Urologie Urologie gynécologique

Dreher, E.; Adam, P.J.; Fischer, B. von und Maurer, H.P.: Uroflowmetrie in der

Gynäkologie 98

Fischer, B. von; Dreher, E.; Adam, P.J. und Maurer, H.P.: Ein neuer Parameter der

Uroflowmetrie in der Gynäkologie 99

Lienhard, P.; Steuble, E. und Eberhard, J.: Veränderungen der Urethraverschluss-

funktion durch Inkontinenzoperationen - Kolporrhaphia anterior, Marshall-

Marchetti, Lyoduraschlinge 101

Bänninger, U.; Reich, P. und Kunz, J.: Interpretation des mit dem Millar ${ }^{\circledR}$ -

Mikrotransducer gemessenen Urethradruckprofils 103

Gysler, R.; Nüesch, A. und Eberhard, J.: Morphologische Veränderungen nach Inkonti nenzoperationen $\quad 105$

Weil, A. et Gaudenz, R.: Apport du test du lange électronique (Urilos) dans Гinvestigation de Tincontinence d'effort 107

Maurer, HP.; Dreher, E.; Adam, P.J. und Fischer, B. von: Behandlung der sensorischen Urge-Symptomatik der Blase mit Cysto-Caps ${ }^{\circledR} \quad 108$

Gaudenz, R.: Der Inkontinenz-Fragebogen mit dem neuen Urge-Score und Stress-Score 110

Senologie Sénologie

Spinelli, A. und Gysler, R.: Zur audiovisuellen Information der Frauen über die

Brustselbstpalpation 114

Brun del Re, R.; Stucki, D. und Torhorst, J.: Ein neues Gerät zur einhändigen

Bedienung der „Tru-Cut”-Biopsienadel. Mamma-Biopsie, Parametrium-Biopsie . 115

Stucki, D.; Brun del Re, R.; Biedermann, K.; Eppenberger, U. und Almendral, A.:

Ergebnisse beim Vergleich der Thermographieresultate mit biochemischen

Parametern 117

Eppenberger, U.; Stucki, D.; Roos, W.; Brun del Re, R.; Biedermann, K. und Almen

dral, A.: Korrelation von Östrogen- und Progesteron-Rezeptoren beim Mamma-

karzinom 119

Welti, S.; Floersheim, Y. und Keller, P.J.: Behandlung hyperprolaktinämischer Zu-

stände mit Lisurid 120

F amilienplanung/S terilität Planning familial/Stérilité

Soyka, E.; Reinhardt, P. und Muehlemann, M.: Erste Erfahrungen mit dem Mini- 
Gravigard 123

Gloor, P.-A. et Janson, I.: Grossesses non désirées sur échec de la contraception orale . 124

Kunz, J.; Gerig, B. und Schreiner, W.E.: Interruptio graviditatis mit Vakuumaspiration oder Uterotomie 125

Index IV

Kunz, J.; Reich, P. und Bänninger, U.: Vergleichende Untersuchung über die abortive Wirkung von extraamnial instilliertem natürlichem und synthetischem ProstaglandinE2 127

Baumann-Christen, W.: Die ambulante laparoskopische Tubenkoagulation in Lokalanästhesie 130

Stoffel, R. und Bretscher, J.: Erfahrungen mit der Ultraschallkontrolle des Follikelwachstums 131

Grob, B.; Hinselmann, M. und Campana, A.: Uterusseptum als seltene Ursache eines

Interruptioversagers 134

Operative Gynäkologie Gynécologie opératoire

Kunz, J. und Genswein, A.: Beitrag zur kurzzeitigen perioperativen Antibiotika-

prophylaxe in der Gynäkologie 136

Schams, H.: Periduralanästhesie bei vaginalen Operationen 139

Gynäkologische Carcinome Cancer gynécologique

Heinzl, S. und Szalmay, G.: Vorbedingungen zur lokalen konservativen Behandlung der

zervikalen intraepithelialen Neoplasie

Pabst, W.; Eggenberger, M. und Engeler, V.: Endokrine Befunde beim Korpuskarzinom 142

Stoffel, R. und Stoll, W.: Die prophylaktischen Untersuchungen beim Korpuskarzinom 144

Boesinger, U.E.; Iklé, F.A.; Szalmay, G. und Haller, U.: Aspirationsbiopsie des Endo-

metriums nach Iklé als Alternative zur Kürettage 146

Schweizerische Gesellschaft für Gynäkologie

Bericht über die Jahresversammlung, 28. bis 30. Juni 1979 in Montreux

Société Suisse de Gynécologie

Compte rendue de ГAssemblée annuelle, 28 au 30 juin 1979 à Montreux Redaktor - Rédacteur:

E. Dreher, Bern 